\title{
Arbeitsbedingungen von Beschäftigten in Betrieben mit ergebnisorientiert gesteuerten Arbeitsformen
}

Es ist bekannt, dass Arbeitsbedingungen mit gesundheitlichen Belastungen zusammenhängen. Sehr viel weniger wissen wir über einen möglichen Zusammenhang zwischen ansteigenden psychischen Belastungen in den Betrieben und den jeweiligen betrieblichen Rahmenbedingungen, wozu vor allem neue, ergebnisorientierte Steuerungsformen zählen. Die repräsentativen Ergebnisse einer bundesweiten Betriebsrätebefragung, die im Rahmen des Projektes PARGEMA im Herbst/Winter 2008/09 unter 1.700 Betriebsräten durchgeführt wurde und diesen Zusammenhang untersucht hat, deuten - besonders für Betriebe mit ergebnisorientiert gesteuerten Arbeitsformen - auf wachsende psychische Belastungen und größeren Arbeitsdruck hin.

\section{Einleitung}

Ansätze zur Reduzierung von psychischen Arbeitsbelastungen können nur dann Erfolg versprechend sein, wenn der betriebliche Kontext im Vorfeld mitberücksichtigt wurde (vgl. etwa Oppolzer 2010). Empirisch allerdings zeigt sich hier eine Forschungslücke, zumal es kaum (quantitativ) empirisches Datenmaterial gibt, das einen Zusammenhang zwischen der Verbreitung psychischer Arbeitsbelastungen und den dazugehörigen Rahmenbedingungen im Betrieb untersucht. Dabei haben sich die betrieblichen Rahmenbedingungen und die Leistungspolitik in den Unternehmen seit den 1990er Jahren grundlegend verändert. Zum Beispiel hat es in den Unternehmen eine vermutlich nie dagewesene Welle von Umstrukturierungen in privatwirtschaftlichen und öffentlichen Betrieben gegeben - ein Tatbestand, der sich natürlich auch auf die Arbeitsbedingungen der Beschäftigten auswirkt (vgl. etwa Kieselbach 2009).

Umstrukturierungen sind unter mehreren Gesichtspunkten für die Entwicklung der Arbeitsbedingungen der Beschäftigten von Bedeutung. Erstens, weil sie in den meisten Fällen aus Rationalisierungsgründen erfolgt sind und die Personaldecke sukzessive haben schrumpfen lassen. In der Praxis bedeutet dies, dass heute weniger Beschäftigte für eine wachsende Aufgabenvielfalt zuständig sind. Zweitens, weil Umstrukturierungen fast immer mit Ängsten und Verunsicherungen einhergehen (Kieselbach 2009). Und drittens, weil sich in vielen Fällen die Organisation von Arbeit verändert hat - Leitbilder wie Flexibilisierung und Subjektivierung von Arbeit nehmen seither eine dominante Rolle in der betrieblichen Arbeitsorganisation ein. Aufgrund von Dezentralisierungsmaßnahmen und damit einhergehenden abgeflachten Hierarchien sind Verantwortung und Kompetenzen von der Ebene des Managements auf die Ebene der Arbeitnehmer verlagert worden. Dies bedeutet für die Beschäftigten, sowohl mit deutlich gestiegener Verantwortung als auch mit zunehmender Komplexität in den Arbeitsinhalten umgehen zu müssen. Eine Reihe dieser so entstandenen Arbeits- und Steuerungsformen zeichnet sich dadurch aus, dass die Leistung der Beschäftigten über das Ergebnis gesteuert wird. Beispiele dafür sind Projektarbeit, ergebnisorientiertes Arbeiten mithilfe von Zielvereinbarungen oder der Einsatz von betrieblichen Kennziffern zur Leistungskontrolle der Beschäftigten.

Für die Beschäftigten können diese Arbeitsformen durchaus mit Hoffnungen verbunden sein, zumal sie die Chance auf ganzheitliche Aufgaben und erweiterte Spielräume beinhalten. In der Praxis zeigt sich jedoch, dass ergebnisorientierte Arbeitsformen für viele Beschäftigte in erster Linie mit psychisch belastendem Arbeits- und Leistungsdruck verbunden sind (Kratzer 2003; Gerlmaier 2004; Pröll/ Gude 2003).

Welche Rolle ergebnisorientierte Arbeitssysteme hinsichtlich des ansteigenden Arbeitsdrucks spielen, soll im Folgenden über Leitfragen thematisiert und analysiert werden:
(1) Verbreitung von ergebnisorientierten Arbeitssystemen: Die Frage stellt sich, ob hier ein branchenbedingtes Phänomen vorliegt, wie es für die IT-Dienstleistungen (Gerlmaier 2004) oder Banken und Versicherungen oft angenommen wird.

(2) Die Ambivalenz in den Auswirkungen auf die Beschäftigten: Ergebnisorientierte Arbeitssysteme können für die Beschäftigten einen wertvollen Beitrag zu einer bereichernden Arbeitswelt leisten. Sie bieten die Chance, dass ganzheitliche Aufgaben und erweiterte Spielräume erzielt werden. Tatsächlich wird die Arbeit von vielen Beschäftigten als herausfordernd und interessant erlebt - und weil die Beschäftigten ihren Arbeitsablauf selbst organisieren können, beinhaltet dieses Steuerungsinstrument ein gesund erhaltendes Potenzial. Die Chancen und das Potenzial dieser ergebnisorientierten Arbeitssysteme können jedoch schnell in psychische Fehlbelastungen und gesundheitliche Gefährdungen übergehen. Etwa dann, wenn die Beschäftigten zur Erreichung ihrer vorgegebenen Ziele (bei allen gewährten Freiheiten) über zu wenige Ressourcen verfügen - sei es aufgrund von zu geringen Zeitpuffern, fehlender Unterstützung durch Kollegen oder Führungskräfte, nicht ausreichender Weiterbildung bei Übertragung neuer Aufgabengebiete, etc.

Ahlers, Elke, Promotionsstipendiatin der Hans-Böckler-Stiftung. Arbeitsschwerpunkte: Betriebs- und Personalräte-Befragung Arbeits- und Gesundheitsschutz. e-mail: elke.ahlers@googlemail.com 
Studien zeigen, dass dies in der Praxis oft der Fall ist (vgl. Dunkel et al. in diesem Heft). Die Arbeitsbedingungen können also von den Beschäftigten als bereichernd und belastend zugleich empfunden werden. Die Ambivalenz in den Arbeitsbedingungen könnte dazu beitragen, dass die Verantwortung für das Erreichen bestimmter Leistungsziele und für den Erhalt der eigenen Gesundheit in Widerspruch zueinander geraten - etwa durch eigenständige Verlängerung der Arbeitszeiten und den Verzicht auf Pausen oder krankheitsbedingte Abwesenheit. Das erschwert die Gestaltung von Arbeit und verlangt nach neuen Ideen und Konzepten.

Bevor die soeben dargestellten Annahmen über die Rolle neuer Arbeits- und Steuerungsformen in den Betrieben mit quantitativen Daten beleuchtet werden, soll die Wahl der Methodik und sodann die Befragung als solche vorgestellt werden (Abschnitt 2). Der Überblick über die Ergebnisse der Befragung (Abschnitt 3) beginnt mit der Bandbreite der Arbeitsbedingungen in den Betrieben, liefert Daten zur Verbreitung von neuen Arbeits- und Steuerungsformen, thematisiert Wirkungen ergebnisorientierter Arbeits- und Organisationsformen auf die Arbeitsbedingungen der Belegschaften und endet mit Effekten für die betriebliche Interessenvertretung. Ein Fazit (Abschnitt 4) rundet den Beitrag ab.

\section{Methodik der PARGEMA/ WSI-Betriebsrätebefragung}

Das Projekt PARGEMA hat über die regelmäßig durchgeführten WSI-Betriebsrätebefragungen ${ }^{1}$ im Herbst/Winter 2008/09 telefonisch 1.700 Betriebsräte interviewt, um aktuelle und umfassende Informationen zu Arbeitsbedingungen der Belegschaften und zu den Rahmenbedingungen im Betrieb zu erhalten. ${ }^{2}$ Für die Entscheidung, projektrelevante quantitative Daten über die WSI-Betriebsrätebefragung zu gewinnen, mussten Vor- und Nachteile dieser Datenquelle abgewogen werden. Die Nachteile liegen vor allem darin, dass naturgemäß nur solche Betriebe in das Befragungssample aufgenommen werden, die über einen Betriebsrat verfügen und damit strukturell den vermutlich ar- beitnehmerfreundlicheren Betrieben zuzuordnen sind. Außerdem kommen die Aussagen zum Belastungsgeschehen in den Belegschaften in gewisser Weise aus zweiter Hand, denn es wurden nicht die Belegschaften selbst, sondern deren Vertreter, die Betriebsräte dazu befragt, welchen Arbeitsbedingungen die Beschäftigten ausgesetzt sind.

Für die WSI-Betriebsrätebefragung spricht, dass Betriebsräte als Bindeglied zwischen Management und Belegschaft fungieren und damit die uns interessierenden betrieblichen Gegebenheiten umfassend und kritisch darstellen können. Auch die Nöte und Belastungen in der Belegschaft haben sie im Blick. Die WSIBetriebsrätebefragung deckt zudem einen großen Teil der Beschäftigtenlandschaft ab, denn ungefähr die Hälfte aller Beschäftigten in Deutschland ist in Betrieben mit Betriebsratsvertretung tätig (Ellguth/ Kohaut 2008). Weil es bei den zu erhebenden Daten im Kern darum geht, den Zusammenhang zwischen betrieblichen Rahmenbedingungen und den Arbeitsbelastungen im Betrieb zu beleuchten - und nicht die Arbeitsbelastungen einzelner Beschäftigter zu erfragen - fiel die Wahl auf die WSI-Betriebsrätebefragungen als Datenerhebungsinstrument.

Im Rahmen des dann durchgeführten Erhebungskonzepts wurden Fragen zur Arbeitsorganisation, zur Leistungssteuerung, zum Führungsverhalten, zu Umstrukturierungen und zur wirtschaftlichen Situation des Betriebs gestellt. Auch Umsetzungsaspekte des betrieblichen und gesetzlichen Arbeits- und Gesundheitsschutzes spielten eine wichtige Rolle.

Die Betriebsräte für die computergestützten Telefoninterviews (CATI) wurden im Vorfeld anhand einer geschichteten Zufallsstichprobe ermittelt. Grundlage für die Stichprobenziehung waren übermittelte Rahmendaten des IAB-Betriebspanels hinsichtlich der Verteilung von Betrieben mit Betriebsräten innerhalb bestimmter Branchen und Größenklassen. Die auf dieser Basis durchgeführten Interviews wurden anschließend nach Branche und Betriebsgrößenklasse gewichtet, sodass die hier vorgestellten Forschungsergebnisse repräsentativ für Betriebe ab 20 Beschäftigte mit Betriebsratsvertretung sind. ${ }^{3}$ Damit lassen sich erstmalig branchen- und betriebsgrößenübergreifende quantitativ-empirische Befunde zu Arbeit und Gesundheit im Kontext betrieblicher
Rahmenbedingungen, wie der Existenz neuer Arbeits- und Organisationsformen, darstellen.

\section{Die Befunde}

\subsection{ARBEITSBEDINGUNGEN UND ARBEITSBELASTUNGEN}

Zunächst soll ein allgemeiner Blick auf die Arbeitsbedingungen der Beschäftigten erfolgen: Danach beurteilt knapp die Hälfte aller befragten Betriebsräte die allgemeinen Arbeitsbedingungen des Betriebes mit gut bis sehr gut (47\%) - also zunächst überraschend positiv. ${ }^{4}$ Unter den positiven Arbeitsbedingungen (Mehrfachantworten) wurde von den Betriebsräten das "eigenverantwortliche Arbeiten“ am häufigsten genannt (55\%). Auch ein gutes Betriebsklima trägt in $40 \%$ der Fälle zu eher guten Arbeitsbedingungen bei. Interessante Herausforderungen durch Projekt- und Teamarbeit war für $29 \%$ der Befragten eine positive Rahmenbedingung im Betrieb. Weniger oft wurden gute Aufstiegsund Qualifizierungsmaßnahmen (21\%), Familienfreundlichkeit (20\%), Anerkennung durch den Arbeitgeber (19\%) sowie gutes Führungsverhalten (18\%) genannt.

Trotz des positiven Gesamteindrucks der Betriebsräte wird der viel zitierte Belastungswandel in den Belegschaften von den Betriebsräten bestätigt (vgl. auch Oppolzer 2010; Ahlers/Brussig 2004). Die psychischen Belastungen der Beschäftigten sind in der Wahrnehmung der meisten Betriebsräte $(79 \%)$ weiter angestiegen wohingegen die körperlichen Belastungen stagnieren (58\%). ${ }^{5}$ Nun soll damit keineswegs behauptet werden, dass körperliche

1 Ziel der WSI-Betriebsrätebefragungen ist es generell, Daten zu Entwicklungen im Betrieb aus Sicht der Betriebsräte zu erhalten (Schäfer 2008).

2 Die Befragung wurde vom infas Institut in Bonn durchgeführt und hatte eine durchschnittliche Interviewlänge von 25 Minuten.

3 Zu detaillierteren methodischen Ausführungen zur PARGEMA/WSI-Befragung siehe Ahlers 2010a.

4 Ein großer Teil (41\%) stuft die Arbeitsbedingungen als "befriedigend" ein. $9 \%$ werten die Arbeitsbedingungen als "ausreichend " und $3 \%$ als "schlecht" bis "sehr schlecht".

5 Allerdings gehen $26 \%$ der Betriebsräte davon aus, dass auch die körperlichen Belastungen wieder ansteigen 
Belastungen in der heutigen Arbeitswelt sekundär wären. Der Fokus liegt in diesem Beitrag allerdings auf den psychischen Fehlbelastungen.

In vielen Untersuchungen wird der wachsende Termin- und Zeitdruck von Beschäftigten hervorgehoben (Bibb/BAuABefragung; DGB-Index Gute Arbeit). Die Ergebnisse der PARGEMA-Betriebsrätebefragung belegen den ansteigenden Terminund Zeitdruck in den Betrieben: In $84 \%$ der deutschen Betriebe gibt es Mitarbeiter, die ständig ${ }^{6}(!)$ unter hohem Zeit- und Leistungsdruck arbeiten. Davon betroffen sind nicht nur einzelne Beschäftigte mit speziellen Aufgaben, sondern durchschnittlich geschätzte $43 \%$ der Belegschaft. Besonders stark leiden demnach Beschäftigte in Dienstleistungsberufen, allen voran in Kreditinstituten und Versicherungen sowie Beschäftigte in den Branchen Verkehr, Nachrichten und Telekommunikation, unter Termin- und Zeitdruck. In diesen Branchen ist nach Einschätzung der Betriebsräte sogar jeder zweite Beschäftigte betroffen. Aber auch in den übrigen Branchen zeigt sich deutlich, dass Zeit- und Leistungsdruck in den Belegschaften weit verbreitet ist. Der hohe Anteil der betroffenen Beschäftigten lässt schon vermuten, dass nicht nur bestimmte Beschäftigtengruppen, wie etwa Führungskräfte oder Vertriebsmitarbeiter, dauerhaft unter Zeitnot und hohem Arbeitsdruck stehen.

Gefragt nach den betroffenen Beschäftigtengruppen, verweist die Mehrzahl der Betriebsräte (76 \%) auf die mittleren Angestellten mit Fachausbildung. Erst an zweiter und dritter Stelle werden Führungskräfte (67\%) und Facharbeiter (60\%) als Betroffene genannt. Der dauerhafte Arbeitsdruck hat also mittlerweile den Kern der Belegschaften erreicht. Als Grund dafür wird von den Betriebsräten am häufigsten eine zu geringe Personaldecke für die hohe Arbeitsbelastung verantwortlich gemacht (84\%). Auch in der oftmals „hohen Eigenverantwortlichkeit der Beschäftigten in der Arbeit" sehen viele einen Auslöser (79 \%). Darüber hinaus gehen drei von vier Betriebsräten davon aus, dass die hohe Abhängigkeit von Kundenvorgaben bei den Beschäftigten auf Dauer Stress verursacht (75\%).

Abbildung 1 zeigt eine Palette an erfragten Arbeitsbelastungen, deren Ausmaß der Betriebsrat für seine Belegschaft auf einer Skala zwischen 1 „sehr stark“ und 6 „überhaupt nicht“ einschätzen sollte. Die

\section{Abb. 1: Die Verbreitung unterschiedlicher Arbeitsbelastungen in}

den Betrieben* - in \% -

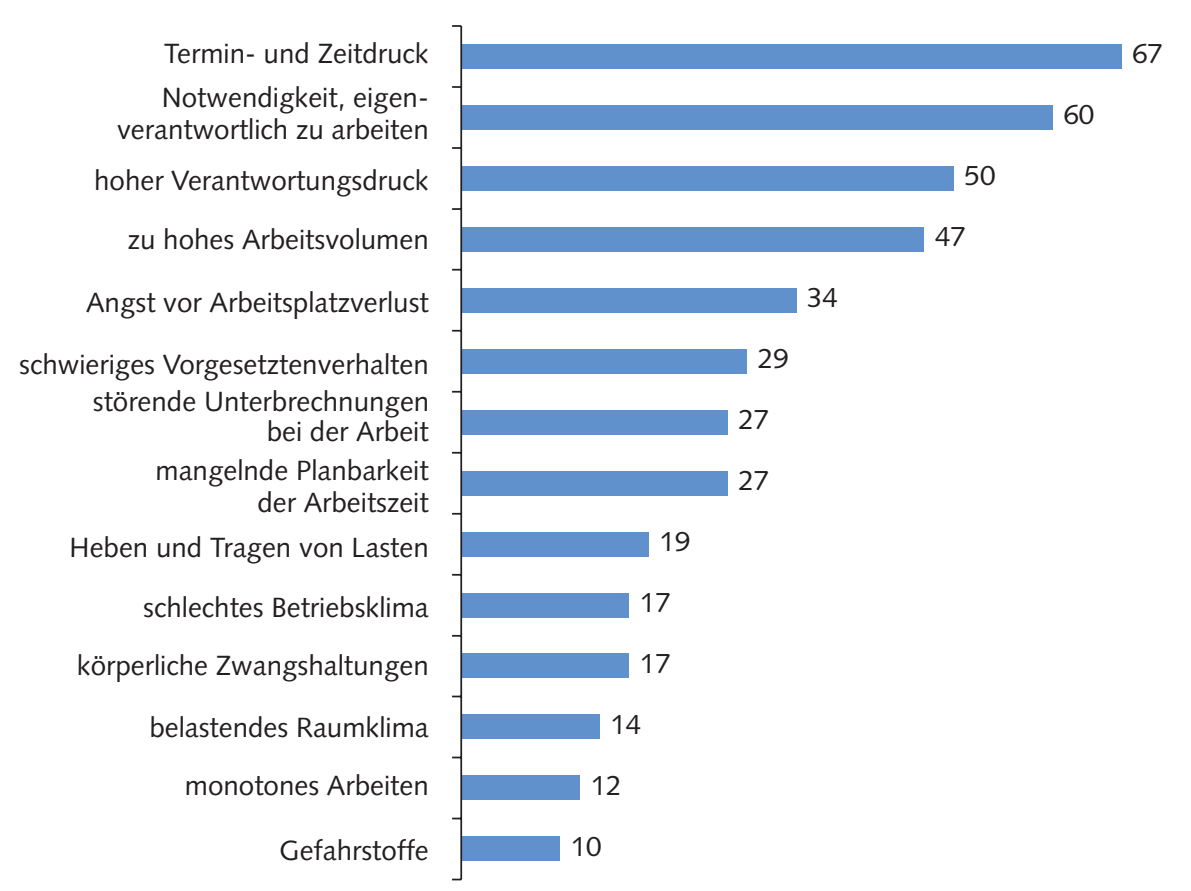

*Folgende Belastungen in der Belegschaft werden von den befragten Betriebsräten als "stark bis sehr stark" wahrgenommen.

Quelle: PARGEMA/WSI-Betriebsrätebefragung 2008/09 zu Arbeitsbedingungen und Gesundheit im Betrieb.

WSI MITTELLUNGEN

errechneten Angaben in der Abbildung 1 spiegeln nur Wertungen wieder, die entweder mit „stark“ oder „sehr stark“ angegeben wurden. Die Werte umfassen also jeweils den Anteil der Betriebe, deren Betriebsräte bestimmte Arbeitsbelastungen ihrer Belegschaft nicht nur beobachten, sondern als herausragend wahrnehmen.

Termin- und Zeitdruck wird von den 1.700 Betriebsräten als die herausragendste Arbeitsbelastung bei den Beschäftigten angegeben (67\%). Eigenverantwortliches Arbeiten, was schon bei den positiven Arbeitsbedingungen genannt wurde, wird zugleich als Belastung gesehen (60\%). Dazu passt der von den Betriebsräten so oft genannte hohe Verantwortungsdruck in den Betrieben (50 \%).

Viele der weiteren aufgelisteten Belastungen - hohes Arbeitsvolumen (42\%), störende Unterbrechungen in der Arbeit (27\%), Vorgesetztenverhalten (29\%) können als Ausdruck von „Arbeitsdruck“ gewertet werden. Zugleich spielt auch die Angst vor Arbeitsplatzverlust (34\%) in vielen Betrieben eine nicht zu unterschätzende Rolle. Die damit verbundene (oft existenzgefährdende) Unsicherheit kann die bestehende Belastungssituation der Beschäftigten noch verstärken.

\subsection{ARBEITSDRUCK UND BETRIEB- LICHE RAHMENBEDINGUNGEN}

Der zu beobachtende Arbeitsdruck in den Belegschaften wird vielfältige Ursachen haben. Eine davon soll hier näher untersucht werden: Im Zuge der erfolgten Umstrukturierungen in den letzten Jahren spielen marktliche Elemente bei der Organisation von Arbeit eine zunehmende Rolle (Sauer et al. 2005). Unstrittig sei, so auch Hirsch-Kreinsen (2003, S. 6), „dass der Wandel von Arbeit in besonderer Weise von Arbeitsformen geprägt ist, die auf eine deutlich erweiterte Ökonomisierung menschlicher Arbeitskraft hinauslaufen. Die Abkehr von starren Formen taylorisierter Arbeit und die Ausweitung des Aufgabenspektrums durch die Reintegration planender und ausführender Tätigkeiten sollen brachliegende Qualifikationen und Leistungsreserven erschließen." Im Kontext dieser Rahmenbedingungen und Managementsysteme haben sich vielfältige neue Arbeits- und Steuerungsformen entwickelt (z.B. kennziffernorientierte

6 Die Interviewer wurden beauftragt, die Betonung auf das Wort "ständig" zu legen. 


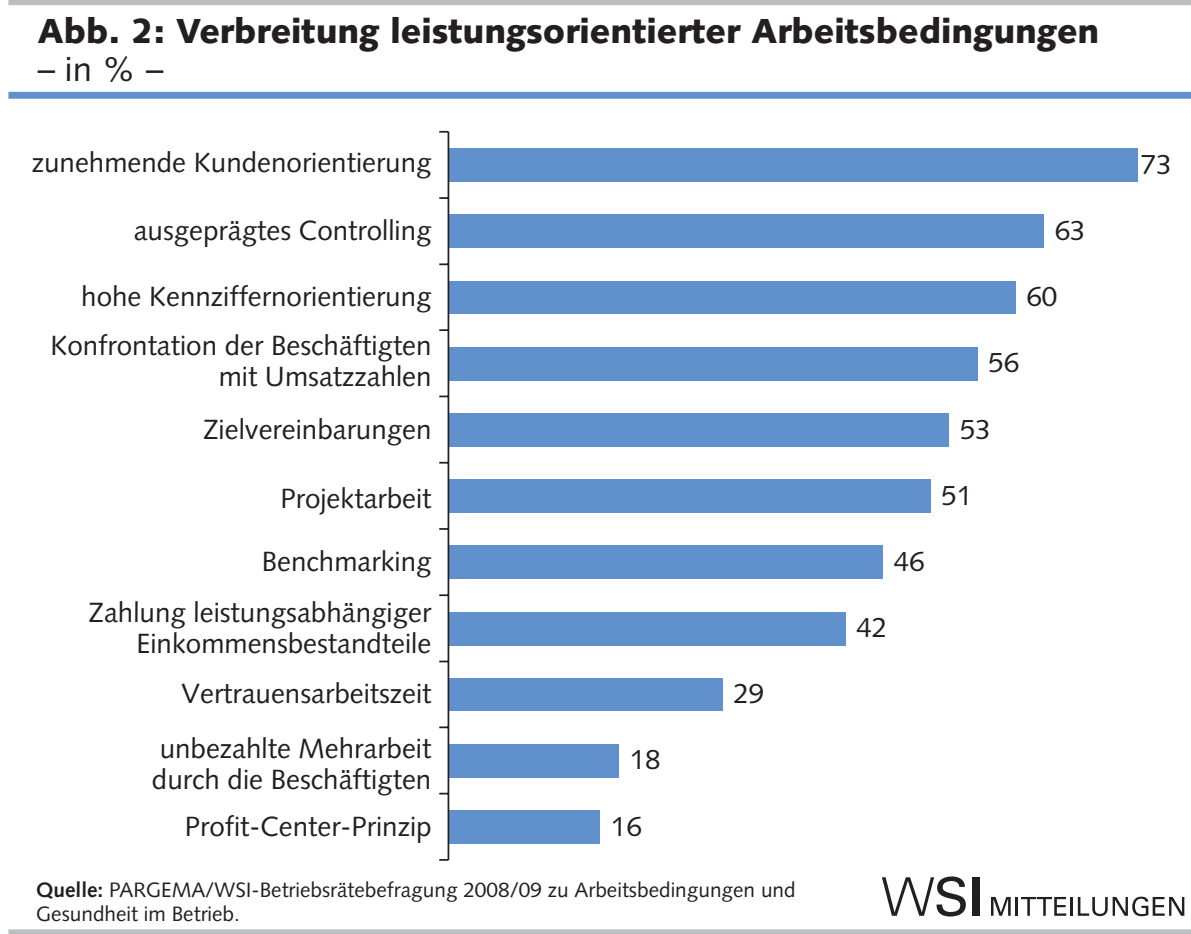

Zielvereinbarungen, Projekt- und Gruppenarbeit, Profit-Center, Benchmarking zwischen Abteilungen). Ihre Gemeinsamkeit liegt in der geforderten Selbstorganisation und der hohen Ergebnisorientierung. Sie unterscheiden sich dagegen von traditionellen Arbeitssystemen im Wesentlichen in ihrer Leistungspolitik: Die Arbeit der Beschäftigten wird nicht mehr direkt in Form detaillierter Arbeitsanweisungen (Detailsteuerung) gesteuert, sondern durch die Vorgabe von Rahmenbedingungen, wie z.B. kennziffernorientierte Zielvereinbarungen, auf die die Beschäftigten selbstständig reagieren sollen. Vor diesem Hintergrund können und müssen die Beschäftigten flexiblere, situationsund entscheidungsabhängige Arrangements treffen, die zugleich hochgradig ambivalent sein können: Auf der einen Seite bekommen sie mehr Freiheiten, aber um den Preis, mehr Eigenverantwortung für Kostenziele und Arbeitsergebnisse zu übernehmen.

Bisher gab es kaum Daten zur Verbreitung ergebnisorientierter Arbeitssysteme, sodass ungewiss war, ob man es mit einem Phänomen einzelner Unternehmen oder Branchen zu tun hat oder ob es eine flächendeckende Tendenz hin zu neuen Steuerungsformen gibt. Um diese Forschungslücke zu schließen, aber auch um einen breiten Eindruck zu sich verändernden betrieblichen Rahmenbedingungen zu erhalten, wurde im Rahmen der Betriebs- rätebefragung zunächst recht breit nach einer sich ändernden Unternehmenskultur und nach unterschiedlichen Arbeits- und Organisationsformen gefragt.

Dabei war zunächst unerheblich, ob alle Beschäftigten oder nur einzelne Abteilungen davon betroffen sind. Zentraler Aspekt bei den erfragten Arbeitsmodalitäten war der Hinweis auf eine ergebnisorientierte Leistungspolitik in den Unternehmen.

Wie Abbildung 2 zeigt, sind Tendenzen hin zu einer stärker ausgeprägten Kundenorientierung $^{7}$ von den Betriebsräten am häufigsten genannt $(73 \%)$. Weiterhin fällt ein Trend zur Kennziffernorientierung im Betrieb auf: So wenden mittlerweile $63 \%$ der Betriebe ein ausgeprägtes Controlling der Betriebsabläufe an. Sechs von zehn Betrieben arbeiten intensiv mit Kennziffern oder anderen betrieblichen Zielgrößen, um Ziele festzulegen. In $53 \%$ der Betriebe werden Mitarbeiter über Zielvereinbarungen geführt. Zielvereinbarungen sind ein typisches Instrument, um die Beschäftigten leistungs- und ergebnisorientiert $\mathrm{zu}$ steuern. In 39 \% der von PARGEMA befragten Betriebe werden alle Beschäftigten oder alle Beschäftigten der mittleren Angestelltenebene über Zielvereinbarungen ${ }^{8}$ geführt. Bei den zu erreichenden Zielen steht inhaltlich fast immer das Erreichen eines konkreten wirtschaftlichen Erfolgs (92\%) oder die Kundenzufriedenheit (88\%) im Vordergrund.
$46 \%$ der Betriebe nutzen Benchmarking-Systeme, um die (kennziffernorientierten) Ergebnisse der Abteilungen oder Teams miteinander vergleichen zu können. Kurzum: Es gibt mittlerweile einen beachtlichen Anteil von Betrieben, in denen Beschäftigte ihre Arbeit auf die Erreichung von Kennziffern - also ergebnisorientiert - ausrichten. Auch bei der Projektarbeit (51 \%) wird eine ergebnisorientierte Leistung gefordert.

Markt- und ergebnisorientierte Arbeits- und Steuerungsformen haben also Einzug in die Betriebslandschaft gefunden. Im Branchenvergleich allerdings werden in den Kreditinstituten und Versicherungen überdurchschnittlich oft solche Steuerungsformen eingesetzt. Die Daten vermitteln einen Eindruck darüber, wie hoch damit der Anteil der Betriebe ist, die (in welchem Umfang auch immer) mit entsprechenden neuen Arbeits- und Steuerungsformen arbeiten. Sie sagen allerdings noch nichts über die Arbeitsbedingungen der Beschäftigten in diesen Betrieben aus.

\subsection{AMBIVALENTE ARBEITS- BEDINGUNGEN BEI NEUEN ARBEITS- UND ORGANISATIONS- FORMEN}

Viele der neuen Arbeits- und Steuerungsformen zeichnen sich durch eine hohe Selbstorganisation der Beschäftigten aus (Sauer et al. 2005). Flexibilität und Eigenverantwortung sind Attribute, die Beschäftigte dafür mitbringen müssen. Betriebe, die ergebnisorientierte Arbeitsformen einsetzen, fallen sowohl hinsichtlich ihrer (von den Betriebsräten beobachteten) positiven als auch hinsichtlich ihrer negativen Arbeitsbedingungen auf. Als Beispiele seien hier Betriebe genannt, die mit Zielvereinbarungen arbeiten oder die Projektarbeit einsetzen. Betriebsräte nennen hier signifikant häufiger „Eigenverantwortung“

7 Die Frage wurde vom Interviewer wie folgt gestellt: „Wie sehr ist die Arbeit in ihrem Betrieb geprägt durch zunehmende Kundenorientierung? Bewerten Sie bitte auf einer Skala von 1 "sehr gut" bis 6 "überhaupt nicht"? $73 \%$ der Betriebsräte antworteten daraufhin mit "stark " bis "sehr stark".

8 Im Branchenvergleich zeigen sich allerdings deutliche Unterschiede: In $81 \%$ aller befragten Kreditinstitute und Versicherungen werden nahezu für alle Beschäftigten Zielvereinbarungen eingesetzt. Im Industriesektor liegt der prozentuale Anteil mit knapp $30 \%$ deutlich darunter. 
Abb. 3: Arbeitsbelastungen beim betrieblichen Einsatz neuer

Arbeits- und Steuerungsformen - in \% -

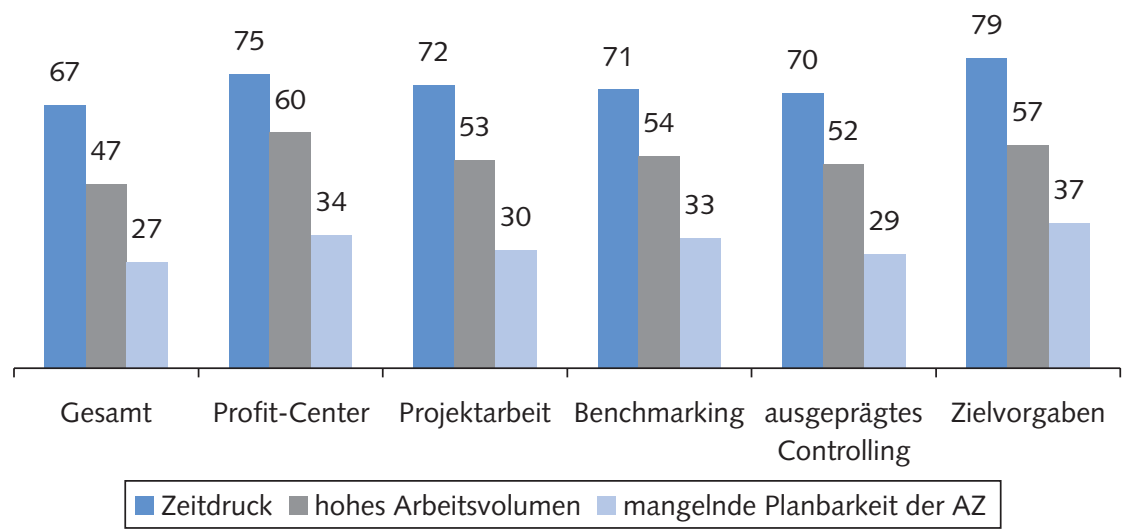

Quelle: PARGEMA/WSI-Betriebsrätebefragung 2008/09 zu Arbeitsbedingungen und Gesundheit im Betrieb.

WSI MITTEILUNGEN

und „interessante Herausforderungen“ als positive Rahmenbedingungen für die Beschäftigten.

Auffällig ist jedoch, dass diese Eigenverantwortung sowohl bei den positiven Rahmenbedingungen als auch bei den Arbeitsbelastungen häufiger genannt wird. Qualitative Studien zeigen, dass ergebnisorientierte Arbeitsformen für die Beschäftigten mit deutlichen Belastungen einhergehen können (Wilde et al. 2010; Kocyba/ Voswinkel 2007). Diese Befunde können hier auf quantitativer Ebene ergänzt und bestätigt werden. Denn die befragten Betriebsräte schätzen auch das Ausmaß der Belastungen (Zeitdruck, Arbeitsvolumen, mangelnde Planbarkeit der Arbeitszeiten) auf die Beschäftigten überdurchschnittlich hoch ein, sobald ein Betrieb eine dieser ergebnisorientierten Steuerungsformen einsetzt.

Sagen noch $67 \%$ aller 1.700 befragten Betriebsräte, dass der Zeit- und Leistungs-

\section{Abb. 4: Beobachtungen von Betriebsräten zum Verhalten der} Beschäftigten - in \% -

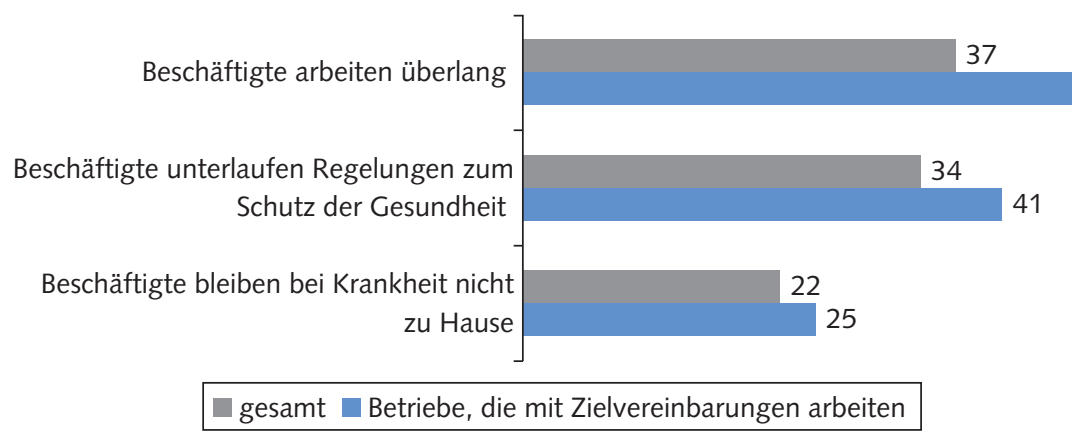

Quelle: PARGEMA/WSI-Betriebsrätebefragung 2008/09 zu Arbeitsbedingungen und Gesundheit im Betrieb. druck an den Arbeitsplätzen die Beschäftigten stark bis sehr stark belasten, so geben dies in den Zielvereinbarungsbetrieben $79 \%$ der Betriebsräte an. In Betrieben, die mit Profit-Centern arbeiten, beläuft sich der Anteil auf $75 \%$. Auch Belastungen durch das hohe Arbeitsvolumen und die geringe Planbarkeit der Arbeitszeit werden deutlich öfter als „stark“ bis „sehr stark“ eingeschätzt als der Durchschnitt (Abbildung 3).

Warum solche Arbeitsbelastungen bei Einsatz dieser Steuerungsformen überdurchschnittlich hoch ausfallen, bleibt zu diskutieren. Branchenphänomene, wie sie etwa für die Banken und Kreditinstitute aufgrund des dortigen hohen Leistungsdrucks und des massiven Einsatzes ergebnisorientierter Arbeitsformen auf der Hand lägen, konnten aufgrund multivariater Analysen ausgeschlossen werden. 1 WSI MitTEILUNGEN

\subsection{BETRIEBLICHE INTERESSEN- VERTRETUNG}

Wenn sich Arbeits- und Leistungskonzepte dermaßen verändern, liegt es nahe, auch die Konsequenzen auf die Regulierung von Arbeitsbedingungen der Beschäftigten zu untersuchen. Das wären einmal Konsequenzen für die betrieblichen Interessenvertretungen und zum anderen Konsequenzen für den Arbeits- und Gesundheitsschutz. Denn das Spannungsverhältnis zwischen interessanten Herausforderungen und Arbeitsdruck könnte auch ein Motiv dafür sein, eigene (gesundheitliche) Interessen zu unterlaufen und bestehende gesundheitliche Schutzregelungen zu umgehen - etwa durch eigenständige Verlängerungen der Arbeitszeit oder Verzicht auf Pausen. Auch traditionelle Konzepte von betrieblichen Interessenvertretungen könnten an ihre Grenzen stoßen, wenn sich aufgrund einer veränderten Leistungspolitik im Unternehmen ein individuelleres Leistungsdenken durchsetzt (u. a. Haipeter 2008).

In Abbildung 4 wird deutlich, dass Regelungen zum Schutze des Arbeitnehmers und seiner Gesundheit häufiger unterlaufen werden, wenn etwa im Betrieb mit Zielvereinbarungen gearbeitet wird:

(1) Hinsichtlich des Arbeitszeitgesetzes berichten $37 \%$ der befragten Betriebsräte, dass Beschäftigte „ihres“ Betriebs überlang (mehr als zehn Stunden am Tag) arbeiten. Wenn im Betrieb durch Zielvereinbarungen ergebnisorientiert gearbeitet wird, sind es mit $47 \%$ der Betriebsräte signifikant mehr, die überlange Arbeitszeiten der Belegschaften beobachten.

(2) $34 \%$ der Betriebsräte geben an, das Beschäftigte Regelungen zum Schutz ihrer Gesundheit (z.B. Pausenregelungen) unterlaufen. Wenn die Belegschaften unter Zielvereinbarungen arbeiten, gibt ein statistisch signifikanter weitaus höherer Teil der Betriebsräte (41 \%) an, dass Beschäftigte Regelungen zum Schutz der Gesundheit missachten.

(3) Und $22 \%$ der Betriebsräte berichten, dass Beschäftigte bei Krankheit nicht zu Hause bleiben. Arbeiten die Beschäftigten wiederum unter Zielvereinbarungen, dann sind es $25 \%$. 
1

Fazit

Die PARGEMA/WSI-Betriebsrätebefragung 2008/09 hatte zum Ziel, die psychischen Arbeitsbelastungen im Kontext von betrieblichen Rahmenbedingungen zu untersuchen. Die Ergebnisse der Befragung bestätigen nicht nur aktuelle Studien zum Anstieg des Arbeitsdrucks in den Betrieben. Sie zeigen zudem, dass sich die zunehmenden psychischen Arbeitsbelastungen der Beschäftigten unabhängig von der Branche- oder der Betriebsgröße darstellen. Neue Arbeits- und Steuerungsformen sind in den Betrieben mittlerweile flächendeckend zu finden. Bei der Betrachtung des zunehmenden Arbeitsdrucks spielen sie - den Analysen der repräsentativen PARGEMA/WSI-Betriebsrätebefragung zufolge - eine wichtige Rolle. Das zeigt sich darin, dass der von den Betriebsräten wahrgenommene Leistungsdruck in den Betrieben umso höher ausfällt, wenn im Betrieb neue Steuerungsformen eingesetzt werden. So lassen sich statistisch signifikante Zusammenhänge zwischen dem Einsatz von Zielvereinbarungen in den Betrieben (für alle Beschäftigten oder die mittlere Angestelltenebene) sowie Projektarbeit und hohem Termin- und Zeitdruck der Beschäftigten nachweisen. Damit werden auch qualitative Untersuchungen in ihren Befunden bestätigt, dass sich ergebnisorientierte Arbeits- und Steuerungsformen auf die Arbeitsbedingungen und Fehlbelastungen der Beschäftigten auswirken (etwa Kratzer 2003; Gerlmaier 2004).
Auch die Ambivalenz der Arbeitsbedingungen bei neuen Arbeits- und Organisationsformen wird durch die Beobachtungen der Betriebsräte deutlich. In Betrieben, die ergebnisorientierte Arbeitsformen einsetzen, werden von den Betriebsräten überdurchschnittlich oft die interessanten Herausforderungen in der Arbeit und die Möglichkeiten für eigenverantwortliches Arbeiten herausgestellt. Eigenverantwortliches Arbeiten wird allerdings sowohl als positive Rahmenbedingung im Betrieb wahrgenommen - zugleich aber auch als Auslöser für den Arbeitsdruck eingestuft. Mit Blick auf den höheren Arbeits- und Leistungsdruck und die überlangen Arbeitszeiten der Beschäftigten lässt sich erahnen, dass in der betrieblichen Wirklichkeit die Potenziale und Chancen ergebnisorientierter Arbeitssysteme in vielen Fällen vergeben werden. Es fehlt den Beschäftigten oft an Zeitpuffern sowie an sozialer Unterstützung von Kollegen oder Vorgesetzten. Auch für erforderlich werdende Nachqualifizierungen fehlt oft die Zeit.

Die hier vorgestellten Beobachtungen aus der betrieblichen Praxis verweisen zudem auf eine veränderte Leistungskultur in den Unternehmen. Dies ist u.a. daran zu erkennen, dass teilweise lang erkämpfte Arbeitszeitregelungen oder Regelungen zum Schutz der Gesundheit überdurchschnittlich oft unterlaufen werden, wenn Beschäftigte ergebnisorientiert arbeiten. Dass es vor diesem Hintergrund zu Spannungen zwischen Betriebsrat und Belegschaft kommen kann, liegt auf der Hand. Bei fast der Hälfte der betroffenen Betriebsräte, deren Beschäftigte unter
Zielvereinbarungen tätig sind, kommt es zu Konflikten mit der Belegschaft, weil Arbeitszeitregelungen missachtet werden. Und bei $41 \%$ der Betriebsräte kommt es zu Konflikten mit der Belegschaft, weil Regelungen zum Schutz der Gesundheit nicht eingehalten werden. Traditionelle Konzepte im Betriebsratshandeln scheinen hiermit empirisch infrage gestellt (Glißmann 2005, 2001; Haipeter 2008) - dabei könnte und müsste Betriebsratsarbeit wegen der wachsenden Bedeutung einer nachhaltigen Arbeitsgestaltung und -regulierung an Bedeutung zunehmen.

Die eingangs erwähnten Umstrukturierungen haben also in vielfältiger Weise zum Anstieg des Arbeitsdrucks in den Belegschaften beigetragen. Erstens, weil sich das Arbeitsvolumen aufgrund von Rationalisierungsmaßnahmen auf immer weniger Beschäftigte verteilt. Zweitens, weil kein Arbeitsplatz mehr sicher erscheint und die Angst vor einem möglichen Verlust des Arbeitsplatzes den ohnehin vorhandenen Arbeitsdruck noch erhöht. Und drittens, weil die im Rahmen von Umstrukturierungsmaßnahmen oft eingeführten marktnahen Arbeitsformen einen weiteren Zugriff auf die menschliche Arbeitskraft bedeuten.

Hinsichtlich der hier dargelegten $\mathrm{Zu}$ sammenhänge zwischen ergebnisorientierten Arbeitsformen und Fehlbelastungen bei den Beschäftigten besteht sicherlich noch erheblicher Forschungsbedarf. Gleichwohl sind Akteure des betrieblichen Gesundheitsschutzes gut beraten, wenn sie die Wirkungsweise von ergebnisorientierten Arbeitsformen auf die Beschäftigten in ihren Gestaltungsansätzen mitberücksichtigen.

\section{LITERATUR}

Ahlers, E. (2010a): Arbeit und Gesundheit in schwierigen Zeiten Ergebnisse der PARGEMA/WSI-Betriebsrätebefragung 2008/09, in: Kratzer, N. et al. (Hrsg.): Partizipatives Gesundheitsmanagement, Frankfurt, S. 331-352, (im Erscheinen)

Ahlers, E. (2010b): Psychische Belastungen und betrieblicher Gesundheitsschutz - wo drückt der Schuh?, in: Pape, K.: Wandel der Arbeit und betriebliche Gesundheitsförderung, Hannover, S. 36-48

Ahlers, E./Brussig, M. (2004): Gesundheitsbelastungen und Prävention am Arbeitsplatz - WSI-Betriebsrätebefragung 2004, in: WSI-Mitteilungen 11, S. 617-624

Ahlers, E./Ziegler, A. (2009): Ein Dammbruch. Zu Umfang und Auswirkungen von Standortverlagerungen in Deutschland, in: Ahlers, E./ Beese, B./Ziegler, A. (Hrsg.): Beschäftigte in der Globalisierungsfalle, Baden-Baden, S. 19-36
Betriebskrankenkassen (BKK) Bundesverband (Hrsg.) (2008): BKK Gesundheitsreport 2008, Essen

Deutscher Gewerkschaftsbund (2009): DGB-Index Gute Arbeit - Der Report 2009. Wie die Beschäftigten die Arbeitswelt in Deutschland beurteilen, Berlin

Ellguth, P./Kohaut, S. (2008): Tarifbindung und betriebliche Interessenvertretung: Aktuelle Ergebnisse aus dem IAB-Betriebspanel 2007, in: WSI-Mitteilungen 9, S. 515-519

Gerlmaier, N. (2004): Projektarbeit in der Wissensökonomie und ihre Auswirkungen auf die Work Life Balance, in: Kastner, M. (Hrsg.): Die Zukunft der Work Life Balance, Kröning, S. 282-304 Glißmann, W. (2001): Betriebliche Interessenvertretung, in: Glißmann, W./Peters, K. (Hrsg.): Mehr Druck durch mehr Freiheit. Die neue Autonomie in der Arbeit und ihre paradoxen Folgen, Hamburg, S. 112-128 
Glißmann, W. (2005): Neue Selbständigkeit in der Arbeit und die Frage der Gesundheit, in: Kuhn, J./Göbel, E./Busch, R. (Hrsg.): Leben um zu arbeiten? Betriebliche Gesundheitsförderung unter biografischem Blickwinkel, Frankfurt a. M., S. 63-89

Haipeter, Th. (2008): Arbeitszeit- und Leistungsregulierung. Neue Regulierungsformen und ihre Folgen für Beschäftigte und Betriebsräte,

in: Dröge, K./ Marrs, K./ Menz, W. (Hrsg.): Rückkehr der Leistungsfrage. Leistung in Arbeit, Unternehmen und Gesellschaft, München, S. $199-219$

Hirsch-Kreinsen, H. (2003): Renaissance der Industriesoziologie?, in: Soziologie 1, S. 5-20

Kieselbach, T. (2009): Gesundheit in der Unternehmensrestrukturierung. Empfehlungen der EU-Expertengruppe HIRES (Health in Restructuring), in: Betriebskrankenkassen (BKK) Bundesverband (Hrsg.): BKK Gesundheitsreport 2009, Berlin, S. 62-64

Kocyba, H./Voswinkel, S. (2007): Krankheitsverleugnung: Betriebliche Gesundheitskulturen und neue Arbeitsformen. Abschlussbericht für die Hans-Böckler-Stiftung, Arbeitspapier der Hans-Böckler-Stiftung 150,

Düsseldorf

Kotthoff, H. (1998): Mitbestimmung in Zeiten interessenpolitischer Rückschritte. Betriebsräte zwischen Beteiligungsofferten und "gnadenlosem Kostendiktat “, in: Industrielle Beziehungen 1, S. 76-100

Kratzer, N. (2003): Arbeitskraft in Entgrenzung. Grenzenlose Anforderungen, erweiterte Spielräume, begrenzte Ressourcen, Berlin

Kratzer, N./Sauer, D. (2005): Flexibilisierung und Subjektivierung von Arbeit, in: SOFI/ IAB/ISF/INIFES (Hrsg.): Berichterstattung zur sozioökonomischen Entwicklung in Deutschland. Arbeit und Lebensweisen, Erster Bericht, Wiesbaden, S. 125-150

Kratzer, N./Menz, W./Nies, S./Sauer D. (2008): Leistungspolitik als Feld „umkämpfter Arbeit“, in: Prokla 150, S. 11-26
Lenhardt, U./Priester, K. (2005): Flexibilisierung - Intensivierung Entgrenzung: Wandel der Arbeitsbedingungen und Gesundheit, in: WSI-Mitteilungen 9, S. 491-497

Oppolzer, A. (2010): Psychische Belastungsrisiken aus Sicht der Arbeitswissenschaft und Ansätze für die Prävention, in: Fehlzeiten-Report 2009, Heidelberg, S. 13-22

Peters, K./Sauer, D. (2005): Indirekte Steuerung - eine neue Herrschaftsform. Zur revolutionären Qualität des gegenwärtigen Umbruchprozesses, in: Wagner, H. (Hrsg.): "Rentier' ich mich noch“" ? Neue Steuerungskonzepte im Betrieb, S. 23-58

Pröll, U./Gude D. (2003): Gesundheitliche Auswirkungen flexibler Arbeitsformen, Schriftenreihe der Bundesanstalt für Arbeit und Arbeitsmedizin, Forschungsbericht 986, Dortmund, Berlin, Dresden

Sauer, D. (2007): Vermarktlichung und Politik - Arbeitspolitik unter den Bedingungen Indirekter Steuerung, in: Peter, G. (Hrsg.): Grenzkonflikte der Arbeit, Hamburg, S. 202-217

Sauer, D./Boes A./Kratzer N. (2005): Reorganisation des Unternehmens, in: SOFI/IAB/ISF/INIFES (Hrsg.): Berichterstattung zur sozioökonomischen Entwicklung in Deutschland. Arbeit und Lebensweisen, Erster Bericht, Wiesbaden, S. 323-350

Schäfer, C. (2008): Die WSI-Betriebsrätebefragung 2007 - Methoden und ausgewählte Ergebnisse, in: WSI-Mitteilungen 6, S. 291-296

Siegrist, J. (2002): Stress. Wie meistern wir die schöne neue Arbeitswelt?, in: GEO 3

Siegrist, J./Dragano, N. (2008): Psychosoziale Belastungen und Erkrankungsrisiken im Erwerbsleben, in: Bundesgesundheitsblatt - Gesundheitsforschung Gesundheitsschutz 3, 305-312

Wilde, B./Dunkel, W./Hinrichs, S./Menz, W. (2010): Gesundheit als Führungsaufgabe in ergebnisorientiert gesteuerten Arbeitssystemen, in: Badura, B. et al. (2010): Fehlzeiten-Report 2009, Heidelberg, S. 147-155 